\title{
THE TRIPLET STATE IN STILBENE cis-trans PHOTOISOMERIZATION
}

\author{
Jack Saltiel*, D. W. L. Chang, E. D. Megarity, A. D. Rousseau, \\ P. T. ShanNon, B. Thomas and A. K.' Uriarte \\ Department of Chemistry, The Florida State University, \\ Tallahassee, Florida 32306, USA
}

\begin{abstract}
Stilbene triplets are further characterized by their interaction with quenchers. A previously proposed model for stilbene triplet decay and quenching, together with rate constants for excitation transfer from indeno[2,1-a]indene triplets to azulene, is used to account for the temperature dependence of the azulene effect on stilbene photoisomerization and is shown to lead to erroneous predictions concerning the lifetime and geometry of stilbene triplets. This conclusion is based on oxygen quenching experiments suggesting a twisted geometry for stilbene triplets in benzene solution with a lifetime of $\sim 120 \mathrm{~ns}$ at $30^{\circ}$.

Azulene quenching experiments show that bromine substitution at the para position of stilbene markedly increases ${ }^{1} \mathrm{~S} \rightarrow \mathrm{T}$ intersystem crossing efficiency while substitution at the meta position does not enhance this process at $30^{\circ}$. These results are consistent with the fact that in contrast to the para position, the meta position is a near node in the highest occupied and lowest unoccupied molecular orbitals of stilbene.
\end{abstract}

\section{INTRODUCTION}

The geometry, lifetime and reactivity of stilbene triplets have to a large measure been inferred from experiments in which the stilbenes function as acceptors or donors of triplet excitation ${ }^{1-3}$. Such approaches will be the subject of the first part of this paper. Knowledge concerning the efficiency of stilbene triplet interactions with a variety of quenchers has been used in determining the extent to which these triplets participate in photoisomerization following direct stilbene excitation ${ }^{1-6}$. In the second part of this paper quenching methods will be applied in determining whether bromine atom substitution influences the efficiency of intersystem crossing in the stilbenes.

\section{THE LIFETIME AND GEOMETRY OF STILBENE TRIPLETS IN SOLUTION}

\section{The interaction of stilbene triplets with quenchers}

Under triplet (sensitized) excitation conditions stilbene photoisomerization has been proposed to occur by decay from twisted triplets, ${ }^{3} \mathrm{p}^{1-3,7}$, which lie lower in energy than cisoid triplets but are nearly isoenergetic with transoid 
triplets, Scheme $1^{1,3}$. The mechanism in Scheme 1 is based in large part on the different responses of the two stilbene isomers as triplet excitation acceptors to changes in the triplet energy of the donor. trans-Stilbene behaves as a classical triplet excitation acceptor, i.e. when a donor triplet has insufficient energy to promote $\mathrm{t} \mathrm{to}^{3} \mathrm{t}$ the decrease in the rate constant for energy transfer, $k_{\mathrm{et}}$, can

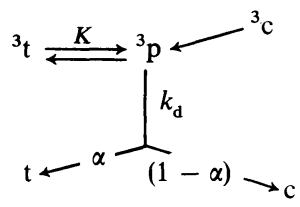

Scheme 1. Mechanism for stilbene triplet decay

be accounted for by assuming that the electronic energy deficiency, $\Delta E_{\mathrm{T}}$, is supplied as an activation energy (equation 1). This suggests that the transoid

$$
\Delta \ln k_{\mathrm{et}}=-\left(\Delta E_{\mathrm{T}}\right) / R T
$$

geometry is at least close to an energy minimum in the triplet state potential energy surface. In contrast, when cis-stilbene is the acceptor, excitation transfer rate constants are much larger than predicted by equation 1 suggesting significant stabilization in the excited state surface as the molecule twists from cisoid geometries towards ${ }^{3} \mathrm{p}^{0}$. This feature of the mechanism explains the inability of molecules with $E_{\mathrm{T}} \approx 57 \mathrm{kcal} / \mathrm{mol}$ (the spectroscopic 'vertical' triplet excitation energy of cis-stilbene) to intercept cisoid triplets ${ }^{3}$. On the other hand the presence of transoid triplets in equilibrium with twisted triplets is suggested by the observation that addition of quenchers having low lying triplets, $E_{\mathrm{T}} \lesssim$ $48 \mathrm{kcal} / \mathrm{mol}$, leads to increased trans $/$ cis photostationary ratios, $([\mathrm{t}] /[\mathrm{c}])_{\mathrm{s}}$,

$$
{ }^{3} \mathrm{t}+\mathrm{Q} \stackrel{k_{\mathrm{q}}}{\longrightarrow} \mathrm{t}+{ }^{3} \mathrm{Q}
$$

presumably due to interaction with transoid triplets (equation 2) whose spectroscopic triplet excitation energy is $49 \pm 1 \mathrm{kcal} / \mathrm{mol}^{1,3}$.

A second quencher type appears to interact with ${ }^{3} \mathrm{p}$. Only one quencher of this type has been reported in the case of stilbenes, di-tert-butyl nitroxide, $\mathbf{N}$, and its presence has been shown to produce a modest increase in $([\mathrm{c}] /[\mathrm{t}])_{\mathrm{s}}$

$$
{ }^{3} \mathrm{p}+\mathrm{N} \longrightarrow \alpha^{\prime} \mathrm{t}+(1-\alpha) \mathrm{c}+\mathrm{N}
$$

(equation $\left.3, \alpha / \alpha^{\prime}=1.11\right)^{12}$. It has been suggested that electron spin exchange with the radical quencher causes vibrational relaxation of the stilbene triplet to the ground state vibrational manifold ${ }^{12}$.

Several versions of potential energy curves for twisting about the central bond in the triplet state of stilbene have been proposed all of which adhere to the requirements of Scheme 1. The differences are in the description of the energetics for twisting from ${ }^{3} t$ to ${ }^{3} \mathrm{p}$. Three possibilities have been considered, the first two having energy minima at ${ }^{3} \mathrm{t}$ and ${ }^{3} \mathrm{p}$ with ${ }^{3} \mathrm{t} \mathrm{lower}^{2,3}$ or higher ${ }^{1}$ in 
energy than ${ }^{3} \mathrm{p}$, and the third, having a single energy minimum at ${ }^{3} \mathrm{t}$ with ${ }^{3} \mathrm{p}$ somewhat higher in energy ${ }^{11,13}$. A fourth possibility which was not meant to account for the results which led to Scheme 1 has ${ }^{3} \mathrm{p}$ significantly lower than either ${ }^{3} \mathrm{t}$ or ${ }^{3} \mathrm{c}$ and is based on molecular orbital calculations ${ }^{14,15}$.

In the following a study of the interaction of stilbene triplets with quenchers will be described which was intended to provide insight concerning the relative energies of ${ }^{3} \mathrm{t}$ and ${ }^{3} \mathrm{p}$. The question whether ${ }^{3} \mathrm{p}$ is higher or lower in energy than ${ }^{3} \mathrm{t}$ is crucial since the answer may provide information bearing on the more important question of whether ground state and triplet energy surfaces touch, cross or avoid crossing at a twisted geometry. It should be noted that with respect to trans ground state molecules the best estimate of the energy content of ${ }^{3} \mathrm{t}$ is $49 \pm 1 \mathrm{kcal} / \mathrm{mol}$ while that of the twisted transition state for thermal cis-trans isomerization is $48 \pm 2 \mathrm{kcal} / \mathrm{mol}^{1}$.

\section{The azulene effect}

We start by adopting the mechanism in Scheme 1 for sensitized stilbene photoisomerization. Using benzophenone as the sensitizer $\left(E_{\mathrm{T}}=68 \mathrm{kcal} /\right.$ $\mathrm{mol})^{19}$, azulene as the quencher $\left(E_{\mathrm{T}}=30 \mathrm{kcal} / \mathrm{mol}\right)^{20}$, and including equation 1 in the mechanism $\left(k_{\mathrm{q}}=k_{\mathrm{az}}\right), k_{\mathrm{az}} / K k_{\mathrm{d}}$ ratios can be obtained either from the response of $([\mathrm{t}] /[\mathrm{c}])_{\mathrm{s}}$ to azulene concentration (equation 4), where $k_{\mathrm{c}}$ and

$$
\left(\frac{[\mathrm{t}]}{[\mathrm{c}]}\right)_{\mathrm{s}}=\frac{k_{\mathrm{c}}}{k_{\mathrm{t}}} \frac{\alpha}{1-\alpha}\left(1+\frac{k_{\mathrm{az}}[\mathrm{Az}]}{\alpha K k_{\mathrm{d}}}\right)
$$

$k_{\mathrm{t}}$ are rate constants for triplet excitation transfer from benzophenone to cis- and trans-stilbene, respectively ${ }^{4}$, or from the response of isomerization quantum yields to azulene (equation 5 ) $^{21}$. The additional azulene effects of

$$
\phi_{\mathrm{t} \rightarrow \mathrm{c}}=(1-\alpha)\left(1+\frac{k_{\mathrm{az}}[\mathrm{Az}]}{K k_{\mathrm{d}}}\right)
$$

internal filtering and quenching of sensitizer triplets do not affect stationary states, and are compensated for in the quantum yield measurements by employing actinometry solutions in which trans-1,2-diphenylpropene whose triplets are not quenched by azulene ${ }^{3}$, is substituted for stilbene ${ }^{21}$. Use of either equation 4 or 5 has been found to give identical $k_{\mathrm{az}} / K k_{\mathrm{d}}$ ratios within experimental uncertainty. The sensitivity of $k_{\mathrm{az}} / K k_{\mathrm{d}}$ to solvent viscosity has played an important role in supporting the notion that intersystem crossing is not a significant decay path when the stilbenes are excited by direct light absorption ${ }^{4}$. Values for these ratios obtained in different solvents at $30^{\circ}$ are shown in Table 1 along with corresponding solvent viscosities. It can be seen that in aprotic solvents the ratios are to a good approximation inversely proportional to solvent viscosity, Figure 1 , as would be expected if $k_{\mathrm{az}}$ were diffusion-controlled and $K k_{\mathrm{d}}$ were solvent independent. In agreement with observations by Liu using anthracenes as quenchers ${ }^{22}$ and by Whitten and Lee using azastilbenes as the donors ${ }^{21}$ anomalously large $k_{\mathrm{az}} / K k_{\mathrm{d}}$ values are obtained in alcoholic media. It has been reasoned that if $k_{\mathrm{az}}$ is diffusion controlled in the more fluid solvents it must surely be diffusion controlled in as viscous 
JACK SALTIEL ET AL.

Table 1. Values of $k_{\mathrm{az}} / K k_{\mathrm{d}}$ at $30^{\circ}$.

\begin{tabular}{lcl}
\hline \multicolumn{1}{c}{ Solvent } & $k_{\mathrm{az}} / K \boldsymbol{k}_{\mathrm{d}}, \boldsymbol{M}^{-1}$ & $\eta, \mathrm{cP}$ \\
\hline$n$-Pentane & 112 & 0.220 \\
Acetonitrile & 88 & 0.329 \\
Toluene & 59 & 0.526 \\
Benzene & 51 & 0.561 \\
Methanol & 106 & 0.510 \\
tert-Butyl alcohol & 85 & 3.316 \\
\hline
\end{tabular}

a solvent as tert-butyl alcohol ${ }^{24}$ and the large $k_{\mathrm{az}} / K k_{\mathrm{d}}$ was tentatively attributed to a marked increase (ninefold in tert-butyl alcohol) ${ }^{4}$ in the effective lifetime of stilbene triplets, $\left(K k_{\mathrm{d}}\right)^{-1}$ in alcoholic solvents ${ }^{4,15}$.

The azulene effect in several solvents has been measured at different temperatures using a miniature merry-go-round apparatus which was initially employed in diene photoisomerization studies ${ }^{25}$. Whitten and Lee's quantum yield method (equation 4$)^{21}$ was used primarily in this work because it

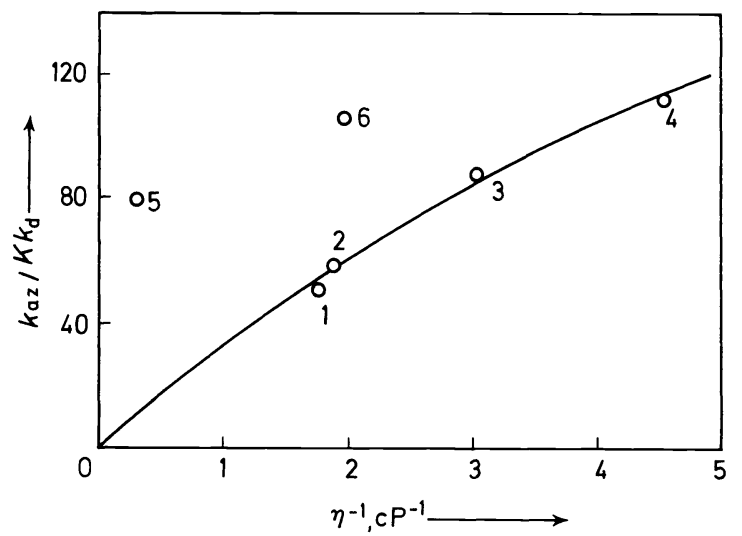

Figure 1. The variation of $k_{\mathrm{az}} / K k_{\mathrm{d}}$ with $\eta^{-1}$ at $30^{\circ}$. Solvents are: benzene 1 , toluene 2, acetonitrile 3, $n$-pentane 4, tert-butyl alcohol 5, methanol 6.

requires shorter irradiation times. Observations in toluene and tert-butyl alcohol, expressed as $k_{\mathrm{az}} / K k_{\mathrm{d}}$ ratios, are plotted versus $T / \eta$ in Figure $2^{26}$. Observations in $n$-pentane and acetonitrile give similar but different curves. The non-linearity of these plots and the fact that data for different solvents fall on different curves appear to suggest that $k_{\mathrm{az}}$ is not the only temperature and solvent dependent quantity but that the effective stilbene triplet lifetime also depends on these variables. The efficiency of exothermic triplet excitation transfer in solution is usually assumed to be diffusion-controlled following a

$$
k_{\text {dif }}=8 R T / 2000 \eta
$$

modified Debye equation (equation 6$)^{27}$. The temperature dependence of stilbene triplet decay rate constants might be extracted from data of the type shown in Figure 2 by assuming $k_{\mathrm{az}}=k_{\mathrm{dif}}$ and applying equation 6 . However, evidence has been presented which suggests that in the less 


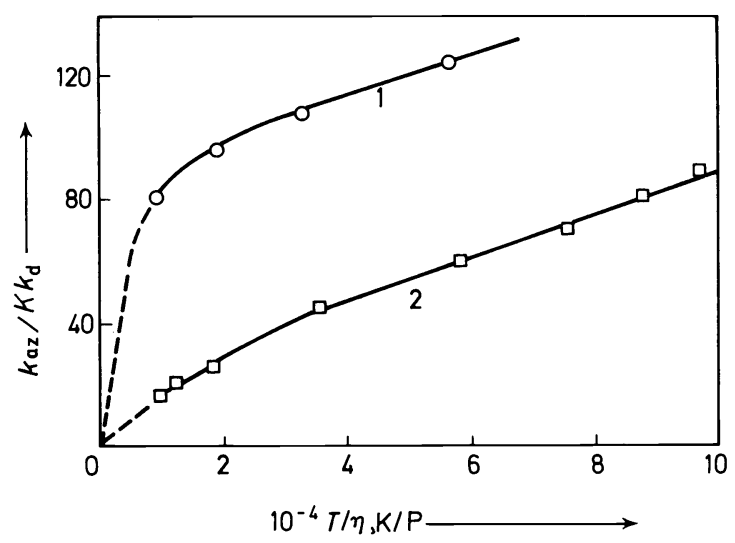

Figure 2. The variation of $k_{\mathrm{az}} / K k_{\mathrm{d}}$ with $T / \eta$ in tert-butyl alcohol (curve 1) and toluene (curve 2).

viscous hydrocarbon solvents usually employed in photochemical studies rates of exothermic triplet excitation transfer can be slower than rates of diffusion ${ }^{24,28}$. It seems desirable, therefore, to have an empirical measure of the $T / \eta$ dependence of $k_{\text {az }}$ instead of relying as before ${ }^{1,4}$ on the Debye equation. Since stilbene triplets are too short-lived to allow their detection by conventional flash-kinetic spectroscopy we have used the rigid stilbene analogue indeno[2,1-a] indene, I, as a model for transoid stilbene triplets (equation 7).

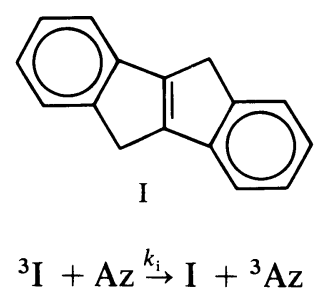

2. The $T / \eta$ dependence of $k_{\mathrm{i}}$

Flash kinetic determination of $k_{\mathrm{i}}$ values has been carried out using a Northern-Precision kinetic flash-photolysis apparatus ${ }^{29}$. Following measurement of the decay characteristics of I triplets, an azulene solution is admitted into the cell via a breakseal and the lifetime of I triplets is measured again. Triple-jacketed cells similar to that described by Jackson and Livingston ${ }^{30}$ were used which allow temperature control by circulation of a transparent liquid (methanol) through the outer jacket. Transient decays were primarily first-order, but second-order decay components were exactly corrected for by using the integrated expression for mixed first- and second-order triplet decay $^{31}$ in conjunction with a general least-squares computer programme developed by $\operatorname{DeTar}^{32}$. The rate constants fall into two main categories. Linear dependence of $k_{\mathrm{i}}$ on $T / \eta$ is observed in toluene and $n$-pentane and non-linear dependence of $k_{\mathrm{i}}$ on $T / \eta$ is observed in tert-butyl alcohol and acetonitrile. The results of the studies in toluene and tert-butyl alcohol which are more complete are plotted in Figure 3. Also shown in Figure 3 is the 
behaviour predicted by the modified Debye equation (equation 6). The fact that linearity is maintained throughout the $T / \eta$ range in toluene suggests strongly that $k_{\mathrm{i}}$ is diffusion-controlled in this solvent. The experimental values are somewhat smaller than those predicted by equation 6 , falling in-

$$
k_{\mathrm{i}}=8 R T / 3750 \eta
$$

stead on a line described by equation 8 . In contrast $k_{\mathrm{i}}$ values in tert-butyl alcohol are larger than those predicted by equation 6 . This is consistent with other observations which suggest that diffusion coefficients in alcohols are double those predicted by the Stokes-Einstein equation upon which equation 6 is based $^{33}$.

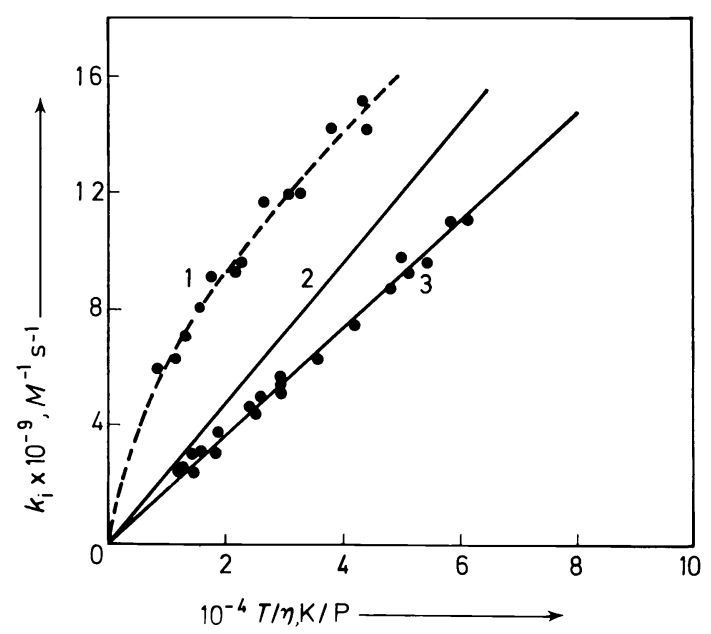

Figure 3. The variation of $k_{\mathrm{i}}$ with $T / \eta$ in tert-butyl alcohol (curve 1) and toluene (line 3). The behaviour predicted by equation 6 is shown by line 2 .

Before applying the above rate constants to the interpretation of the stilbene-azulene data it is of interest to consider these different variations of $k_{\mathrm{i}}$ with $T / \eta$ in toluene and tert-butyl alcohol. Non-linearity of the type exhibited by the tert-butyl alcohol results in Figure 3 has been attributed to a decrease of the rate constants for exothermic triplet excitation transfer below those expected for a diffusion-controlled process as the viscosity of the medium drops below $3 \mathrm{cP}^{24}$. Thus, the curved line could simply reflect a large fraction of ineffective indeno[2,1-a]indene triplet-azulene encounters at the higher temperatures. This possibility seems unattractive for two reasons. First, more pronounced curvature, instead of linearity, would be expected in toluene where much lower viscosities are attained and secondly, most of the observed rate constants in tert-butyl alcohol are larger than would be predicted even if the left hand side of equation 5 were multiplied by two. Another possibility which also seems remote can be considered. Based on its absorption spectrum $^{34}$ and the weak emission spectra which were recently reported ${ }^{20}$, the lowest azulene triplet and singlet excitation energies are 30 and $39 \mathrm{kcal} /$ mol, respectively. Both of these are substantially lower than the trans-stilbene 


\section{THE TRIPLET STATE IN STILBENE cis-trans PHOTOISOMERIZATION}

triplet energy and triplet-singlet excitation transfer by dipole-dipole interaction could in principle compete with diffusion-controlled triplet-triplet excitation transfer. While triplet-singlet excitation transfer is not usually observed in solution its occurrence has been proposed in the benzophenoneperylene system ${ }^{35,36}$ and in the acetone-9,10-dibromoanthracene system, where the presence of the heavy atom substituents appears to be crucial ${ }^{37,38}$. An important criterion for establishing the dipole-dipole nature of the excitation transfer process in the benzophenone-perylene system was the finding ${ }^{36}$ that in agreement with theory ${ }^{39}$, the probability of transfer is proportional to $(T / \eta)^{\frac{3}{4}}$. Interestingly, a plot of $k_{\mathrm{i}}$ in tert-butyl alcohol versus $(T / \eta)^{\frac{3}{4}}$ is linear, possibly suggesting that triplet-singlet excitation transfer is the dominant process in this solvent. If this possibility is tentatively accepted the data in Figure 3 suggest that the nature of the interaction between I and azulene is solvent dependent resulting in triplet-triplet energy transfer in toluene and triplet-singlet energy transfer in tert-butyl alcohol. This conclusion would render the claim that triplet-singlet excitation transfer does not occur in the benzophenone-perylene system ${ }^{39}$ less secure since the $(T / \eta)^{\frac{3}{4}}$ dependence was observed in trichlorotrifluoroethane ${ }^{36}$, while the absence of the dipole-dipole interaction is claimed for benzene solutions ${ }^{40}$. Since the $\mathrm{S}^{1} \leftarrow \mathrm{S}^{0}$ transition in azulene is rather weak ${ }^{34}$ the notion of triplet-singlet excitation transfer in our system does not seem very attractive and we are investigating the third possibility that the curvature of the tert-butyl alcohol data in Figure 3 reflects a small but real deviation from the theoretical prediction of equation 6 for diffusion-controlled triplet excitation transfer.

\section{Use of $k_{\mathrm{i}}$ values in the (mis)interpretation of the azulene-stilbene observations}

A single triplet excitation transfer rate constant from a stilbene to azulene has been measured directly. The donor was 4-nitro-4'-methoxystilbene, nanosecond laser flash-kinetic spectroscopy was employed and the measurement was made in benzene at room temperature ${ }^{5}$. The value of $k_{\mathrm{az}}=6.0 \times$ $10^{9} \mathrm{M}^{-1} \mathrm{~s}^{-1}$ observed in this system is comfortably close to our value of $k_{1}=6.7 \times 10^{9} M^{-1} \mathrm{~s}^{-1}$ measured in benzene at $23^{\circ} \mathrm{C}^{41}$, suggesting that substitution of the $k_{\mathrm{i}}$ values for $k_{\mathrm{az}}$ in the $k_{\mathrm{az}} / K k_{\mathrm{d}}$ ratios should give the temperature dependence of $K k_{\mathrm{d}}$. Thusly lulled into a false security we proceed to calculate effective stilbene triplet lifetimes, $\left(K k_{\mathrm{d}}\right)^{-1}$, ranging from 4.8$8.7 \mathrm{~ns}$ in toluene $\left(+70^{\circ}\right.$ to $\left.-50^{\circ}\right)$ and from $7.7-14.4 \mathrm{~ns}$ in tert-butyl alcohol $\left(80^{\circ}\right.$ to $\left.30^{\circ}\right)$. While the variations in $K k_{\mathrm{d}}$ could be due in part to activation energies associated with $k_{\mathrm{d}}$, the rate constant for intersystem crossing from ${ }^{3} \mathrm{p}$, there are instances where reasonable interpretations of data are obtained by assuming that ${ }^{1} \mathrm{~S} \rightarrow \mathrm{T}$ or ${ }^{1} \mathrm{~T} \rightarrow \mathrm{S}$ intersystem crossing rate constants are temperature independent ${ }^{25,42,43}$. Tentatively assigning the temperature dependence in $K k_{\mathrm{d}}$ entirely to changes in $K$ and substituting the thermodynamic relationship for the equilibrium constant gives equation 9 , where

$$
\log k_{\mathrm{d}} K_{.}=-\frac{\Delta H}{2.303 R T}+\frac{\Delta S}{2.303 R}+\log k_{\mathrm{d}}
$$

$\Delta H$ and $\Delta S$ are the enthalpy and entropy differences between ${ }^{3} \mathrm{t}$ and ${ }^{3} \mathrm{p}$. Plots of the toluene and tert-butyl alcohol data, Figure 4, show satisfactory adherence to equation 9 , giving $\Delta H$ values in the two solvents of 0.8 and 


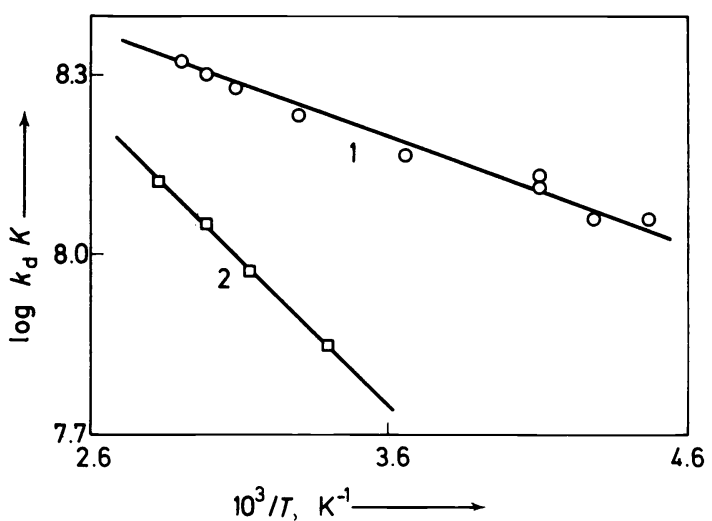

Figure 4. The temperature dependence of estimated $k_{\mathrm{d}} K$ values in toluene (line 1$)$ and in tertbutyl alcohol (line 2).

$2.7 \mathrm{kcal} / \mathrm{mol}$, respectively. The implication is that twisted triplets lie somewhat higher in energy than transoid triplets and that the energy difference is larger in tert-butyl alcohol. Consistent application of the model suggests further than the abnormally high $k_{\mathrm{az}} / K k_{\mathrm{d}}$ ratios in tert-butyl alcohol can be attributed in part to a decrease in the equilibrium constant $K$ which overpowers a substantial increase in $k_{\mathrm{d}}$ suggested by the intercepts of the lines in Figure 4.

\section{The quenching of ${ }^{3} \mathrm{p}$, or the fly in the ointment}

A plausible interpretation of data has been presented indicating a transoid preferred geometry for stilbene triplets in solution. However, in contrast to azulene and several other quenchers which appear to deactivate ${ }^{3} t$ during the excitation transfer process causing more stilbene triplets to decay to the trans isomer (equation 2$)^{1}$, use of di-tert-butyl nitroxide has been shown to reduce stilbene triplet lifetimes, modestly favouring decay to the cis isomer during the deactivation process (equation 3$)^{12}$. To account for the apparent anomaly of quenching of transoid triplets in some cases and of twisted triplets in another it was initially suggested that the free radical quencher forms a complex with stilbene triplets in which ${ }^{3} \mathrm{p}$ geometries are more favoured than normal ${ }^{12 a}$. This proposal was later abandoned in favour of the suggestion that the radical interacts particularly efficiently with stilbene triplets because the ${ }^{1} \mathrm{~T}-{ }^{0} \mathrm{~S}$ energy gap is very small at the equilibrium geometry (presumably ${ }^{3} \mathrm{p}$ ) of the triplet ${ }^{12 b}$. This suggestion is obviously in conflict with the interpretation of the temperature dependence of the azulene effect given above. It must nonetheless be given serious consideration since it can be reasonably argued that the availability of an exchange quenching mechanism not involving triplet excitation transfer allows di-tert-butyl nitroxide to interact with stilbene triplets in their preferred twisted geometry while azulene and other triplet excitation acceptors can interact with stilbene triplets only when large electronic ${ }^{1} \mathrm{~T}-{ }^{0} \mathrm{~S}$ energy gaps are achieved in transoid configurations.

There are two other reasons for concern regarding the validity of the 
assumptions made in the preceding section. First, substitution of $k_{\mathrm{i}}$ for $k_{\mathrm{az}}$ leads to predicted stilbene triplet lifetimes approximately an order of magnitude shorter than the lifetime of a weak transient absorption at $360 \mathrm{~nm}$ observed in benzene solutions of trans-stilbene following excitation with a radiolysis pulse of $25 \mathrm{~ns}$ duration ${ }^{44}$. This transient has been assigned to stilbene triplets and its lifetime ( $110 \pm 10 \mathrm{~ns}$ at room temperature $)$ is in good agreement with lifetimes determined by laser flash-kinetic spectroscopy for several nitrostilbene triplets ${ }^{5}$. Secondly, the efficient quenching of nitrostilbene triplets by oxygen has been measured directly and has been found not to alter $([\mathrm{t}] /[\mathrm{c}])_{\mathrm{s}}$ for these stilbenes ${ }^{5,6}$. Prompted by these observations we have examined the effect of oxygen on the photoisomerization of the stilbenes ${ }^{45}$. The results account for the lifetime discrepancy and require modification of the stilbene triplet decay and quenching mechanisms shown in Scheme 1 and equation 2.

\section{The oxygen effect}

Photostationary trans/cis stilbene compositions were determined for the benzophenone-sensitized photoisomerization in benzene and tert-butyl alcohol as a function of azulene concentration in degassed solution and in the presence of air and oxygen. Excitation was at $366 \mathrm{~nm}$ at $30^{\circ}$ and analyses were by g.l.p.c. as previously described ${ }^{2}$. Air or oxygen were solvent saturated and bubbled through the irradiated solutions. The same results were obtained for solutions which were open to the air and solutions through which a constant air flow was maintained. No significant changes in azulene concentration were detected by u.v. analyses. As for degassed solutions plots of $([\mathrm{t}] /[\mathrm{c}])_{\mathrm{s}}$ ratios obtained in the presence of oxygen versus azulene are linear with the only difference being a strong attenuating oxygen effect on the

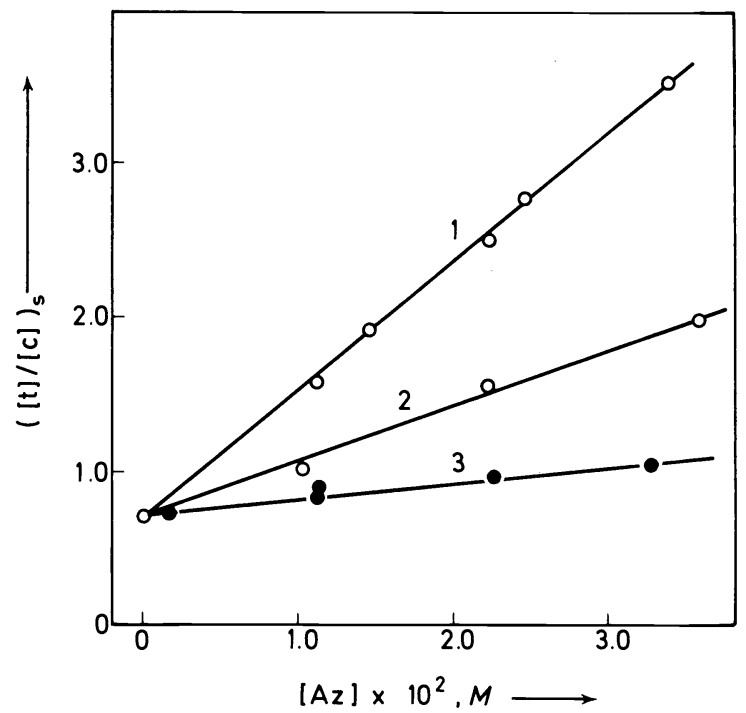

Figure 5. The effect of azulene on the benzophenone-sensitized photoisomerization of the stilbenes at $30^{\circ}$ in benzene: degassed, line 1 ; in the presence of air, line 2 ; in the presence of oxygen, line 3. 
JACK SALTIEL ET AL.

Table 2. Attenuation of the azulene effect by oxygen alcohol

\begin{tabular}{crrcrcc}
\hline & \multicolumn{3}{c}{ Benzene } & \multicolumn{3}{c}{ tert-Butyl alcohol } \\
Conditions & $r$ & $i$ & {$\left[\mathrm{O}_{2}\right], M \times 10^{3} \dagger$} & \multicolumn{1}{c}{$r$} & $i$ & {$\left[\mathrm{O}_{2}\right], M \times 10^{3} \ddagger$} \\
\hline Degassed & 120 & 0.69 & 0 & 201 & 0.77 & 0 \\
Air & 51 & 0.70 & $1.6_{2}$ & 80 & 0.71 & 1.7 \\
$\mathrm{O}_{2}$ & 15 & 0.70 & $7.7_{4}$ & 35 & 0.69 & 8.7 \\
\hline
\end{tabular}

$\dagger$ J. E. Jolley and J. H. Hildebrand, J. Amer. Chem. Soc. 80, 1050 (1957).

‡ Estimated from data for other alcohols in F. Fischer and G. Pfleiderer, Z. anorg. Chem. 124, 61 (1922), cf. J. Livingston, R. Morgan and H. R. Pyne, J. Phys. Chem. 34, 2045 (1930).

slopes of the lines. Data obtained in benzene are plotted in Figure 5. Slope/ intercept ratios, $r$, and the intercepts, $i$, of the lines are shown in Table 2.

The oxygen attenuation effect on the slope of the azulene plots suggests strongly that oxygen interacts with stilbene triplets, thereby decreasing their lifetime. The common intercepts of the sets of lines in each solvent show that the oxygen quenching interaction does not alter the trans/cis decay ratio of the quenched stilbene triplet. The simplest explanation for this result is that oxygen interacts with twisted triplets as shown in non-vertical quenching step

$$
{ }^{3} \mathrm{p}+{ }^{3} \mathrm{O}_{2} \stackrel{k_{\mathrm{ox}}}{\longrightarrow} \alpha \mathrm{t}+(1-\alpha) \mathrm{c}+{ }^{1} \mathrm{O}_{2}
$$

10. A close analogy for this process is provided by the quantum chain carrying step in the photoisomerization of $1,3-$ dienes ${ }^{46,47}$. Since oxygen should also be an efficient quencher of transoid triplets, the important conclusion is that almost all stilbene triplets must be present in twisted geometries in solution. It follows that, contrary to the mechanism assumed above (equation 2), the azulene effect must also have its origin in the interaction of twisted triplets with azulene. These conclusions are incorporated in the mechanism shown in Scheme 2.

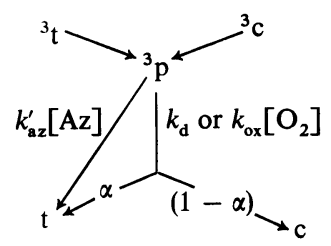

Scheme 2. Modified mechanism for stilbene triplet decay and interaction with quenchers

Accordingly, equation 4 is replaced by photostationary expression 11 . The

$$
([\mathrm{t}] /[\mathrm{c}])_{\mathrm{s}}=\left(k_{\mathrm{c}} / k_{\mathrm{t}}\right)\left(\frac{\alpha}{1-\alpha}\right)\left(1+\frac{k_{\mathrm{az}}^{\prime}[\mathrm{Az}]}{\alpha\left(k_{\mathrm{d}}+k_{\mathrm{ox}}\left[\mathrm{O}_{2}\right]\right.}\right)
$$

lifetime of stilbene triplets, now expressed as $\tau=k_{\mathrm{d}}^{-1}$, can be estimated from the slope/intercept ratios in Table 2 using equation 12, where $r_{0}$ is the

$$
\tau=\left(r_{0}-r\right) / r k_{\mathrm{ox}}\left[\mathrm{O}_{2}\right]
$$


slope/intercept ratio for degassed solutions. Using the benzene data in Table 2 and assuming $k_{\mathrm{ox}}=7.0 \times 10^{9} \mathrm{M}^{-1} \mathrm{~s}^{-1}$, the known value of the rate constant for $p$-nitrostilbene quenching by oxygen ${ }^{5,6}$, values for $\tau$ of 119 and $129 \mathrm{~ns}$ are obtained from air-saturated and oxygen-saturated experiments, respectively. These lifetimes are in excellent agreement with the values obtained in the pulse radiolysis experiments ${ }^{44}$, and since $k_{\mathrm{az}}=\alpha r_{0} / \tau$ they give $k_{\mathrm{az}} \simeq 4.0 \times 10^{8} \mathrm{M}^{-1} \mathrm{~s}^{-1}$, well below the corresponding value of $k_{\mathrm{i}}$. The tert-butyl alcohol data cannot be interpreted quantitatively since neither $k_{\text {ox }}$ nor $\left[\mathrm{O}_{2}\right]$ is known in this solvent. Reasonable estimations of these quantities give $\tau=200 \pm 40 \mathrm{~ns}$ at $30^{\circ}$ suggesting that the large $r_{0}$ observed is not due primarily to an increase in stilbene triplet lifetime as had been previously suggested ${ }^{4,21-23}$, but to a larger $k_{\mathrm{az}}^{\prime}$.

The unsuitability of indeno[ $2,1-\mathrm{az}]$ indene as a model for stilbene triplets provides further support for marked departure of the equilibrium conformation of stilbene triplets from transoid geometry. A corollary to this proposition is the conclusion that in its quenching interaction di-tert-butyl nitroxide ${ }^{12}$ provides a much better probe for the geometry of unperturbed stilbene triplets than does azulene. Upward revision of the stilbene triplet lifetime to $\tau \simeq 120 \mathrm{~ns}$ allows re-evaluation of the rate constant $k_{\mathrm{n}}$ for this process in benzene. Inclusion of equation 3 in Scheme 2 leads to photostationary expression 13. From

$$
([\mathrm{c}] /[\mathrm{t}])_{\mathrm{s}}=\left(k_{\mathrm{t}} / k_{\mathrm{c}}\right)\left(\frac{(1-\alpha)+\left(1-\alpha^{\prime}\right) k_{\mathrm{n}} \tau[\mathrm{N}]}{\alpha+\alpha^{\prime} k_{\mathrm{n}} \tau[\mathrm{N}]}\right)
$$

equation 13 and the data in reference 12 a value of $k_{\mathrm{n}} \tau \simeq 110 \pm 20^{\circ} M^{-1}$ (equivalent to the value of $k_{\mathrm{n}} \tau \alpha^{\prime} / \alpha=100+20$ given in reference 12a) is obtained from which $k_{\mathrm{n}} \simeq 9 \times 10^{8} M^{-1} \mathrm{~s}^{-1}$ can be calculated. This rate constant is not much larger than estimated rate constants for the quenching of rigid planar aromatic hydrocarbon triplets by $\mathrm{N}$ (compare with $k=6.4$ $\times 10^{8} M^{-1} \mathrm{~s}^{-112 \mathrm{~b}, 48}$ for phenanthrene triplet quenching) and suggests that the efficiency of di-tert-butyl nitroxide quenching interactions is not very sensitive to the geometry of the quenched triplet as had been supposed.

\section{Reinterpretation of the azulene effect}

Although as proposed in Scheme 2 the azulene effect probably has its origin in the interaction of ${ }^{3} \mathrm{p}$ triplets with azulene a more detailed mechanism is required in order to account for (1) strong dependence of $k_{\mathrm{az}}^{\prime}$ on solvent viscosity as indicated by the data in Table 1 despite the fact that $k_{\mathrm{az}}^{\prime}<0.1 k_{\mathrm{dif}}$ in benzene, (2) the fact that the quenching interaction gives only trans ground state molecules, and (3) the observation of $k_{\mathrm{az}}^{\prime} \simeq k_{\mathrm{dif}}$ for 4-nitro-4'-methoxystilbene although oxygen experiments indicate a twisted equilibrium geometry for this stilbene also ${ }^{5,6}$. Such an interpretation of the azulene effect is given in equations 14-16, where brackets designate exciplexes or

$$
\begin{aligned}
& { }^{3} \mathrm{p}+\mathrm{Az} \stackrel{k_{14}}{\rightleftarrows}[\mathrm{pAz}] \\
& { }^{3}[\mathrm{pAz}] \stackrel{k_{15}}{\rightleftarrows}[\mathrm{tAz}] \\
& { }^{3}[\mathrm{tAz}] \stackrel{k_{16}}{\rightarrow} \mathrm{t}+{ }^{3} \mathrm{Az}
\end{aligned}
$$


encounter complexes. It follows that $k_{\mathrm{az}}^{\prime}$ in equation 11 is replaced by the expression in equation 17, which reduces to equation 18 if it is assumed that

$$
\begin{gathered}
k_{\mathrm{az}}^{\prime}=\frac{k_{14} K_{15}\left(k_{16} / k_{-14}\right)}{\left(k_{16} K_{15} / k_{-14}\right)+k_{16} / k_{-15}+1} \\
k_{\mathrm{az}}^{\prime}=k_{\mathrm{dif}} K_{15} /\left(1+K_{15}\right)
\end{gathered}
$$

$k_{14}, k_{-14}, k_{16}$ are diffusion-controlled, and that equilibration step 15 is faster than diffusional separation of the donor-acceptor pair. Examination of equation 18 shows that the proposed mechanism accounts for the strong dependence of $k_{\mathrm{az}}^{\prime} \tau$ values on solvent viscosity (Table I). It is important to note here that the alternative mechanism in which step 15 is the rate-determining process leading to excitation transfer, i.e. $k_{-15}=0$, predicts $k_{\mathrm{az}}^{\prime}$ to be approximately proportional to $k_{15}$ thus failing to predict solvent viscosity dependence for $k_{\mathrm{az}}^{\prime}$.

A value of $K_{15} \simeq 0.04$ at $30^{\circ}$ in benzene can be calculated using equation 18 by assuming $\tau=120 \mathrm{~ns}$ and $k_{\mathrm{dif}}=10^{10} \mathrm{M}^{-1} \mathrm{~s}^{-1}$ (see toluene data in Figure $3, T / \eta=5.4 \times 10^{4} \mathrm{~K} / \mathrm{P}$ ). An approximate value of $K_{15} \simeq 0.1$ at $30^{\circ}$ can be calculated from the tert-butyl alcohol data possibly suggesting a somewhat larger stability for the azulene- ${ }^{3} \mathrm{t}$ complex in the more polar solvent. It can also be seen from equation 18 that $k_{\mathrm{az}}^{\prime}$ will approach $k_{\mathrm{dif}}$ in systems for which $K_{15}$ is large (e.g. $>5$ ). It appears that 4-nitro-4'-methoxystilbene represents such a system and it is possible to imagine that in an exciplex intermediate between the triplet state of this polar stilbene molecule and a planar acceptor like azulene more stabilization is attained when the stilbene partner assumes nearly planar transoid geometries.

The temperature dependence of $K_{15}$ in toluene can be estimated from the data in Figures 2 and 3 assuming that $k_{\mathrm{i}}=k_{\mathrm{dif}}$ and that the lifetime of stilbene triplets, $\tau=120 \mathrm{~ns}$, is temperature independent in fluid solutions. A logarithmic plot of $K_{15}$ values obtained in this way versus $T^{-1}$, Figure 6, gives $\Delta H_{15}=-0.81 \mathrm{kcal} / \mathrm{mol}$ and $\Delta S_{15}=-9.1$ e.u. Since even a small tempera-

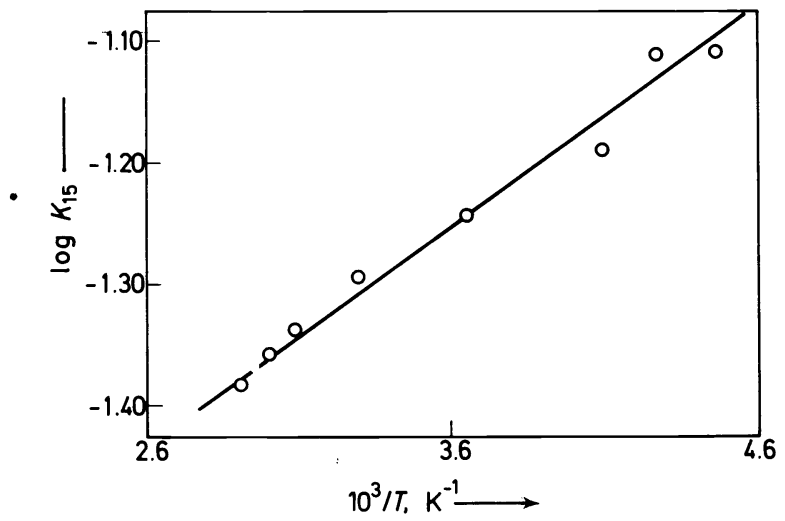

Figure 6. The temperature dependence of estimated $K_{15}$ values in toluene. 
ture dependence in $\tau$ could invalidate this analysis of the calculated $K_{15}$ values, it is presented here primarily because it seems instructive. According to Figure 6 the stilbene triplet-azulene pair has a higher entropy and enthalpy content when the stilbene partner assumes twisted geometries. Thus, it appears that enthalpy favours ${ }^{3} t$ geometries while entropy favours ${ }^{3} p$ geometries in the encounter complex and that entropy wins out over the entire temperature range employed in our experiments. If, furthermore, it is tentatively assumed that azulene does not greatly perturb the energetics of the ${ }^{3} \mathrm{t} \rightleftarrows{ }^{3} \mathrm{p}$ interconversion the same enthalpy-entropy conflict would apply to free stilbene triplets as well. This information can be crucial to the construction of potential energy curves as a function of torsional angle about the central bond, because potential energy differences are best approximated by enthalpy rather than by free energy changes. It is, therefore, quite plausible that the potential energy is lowest for the ${ }^{3} t$ geometry, as had been inferred from the functioning of the stilbenes as acceptors of triplet excitation, but that the free energy at the higher temperatures is lowest for ${ }^{3} \mathrm{p}$ and hence the equilibrium geometry of the stilbene triplets corresponds to ${ }^{3} \mathrm{p}$. At lower temperatures (below $90 \mathrm{~K}$ if we assume that $\Delta H_{15}$ and $\Delta S_{15}$ can be applied to the stilbene triplet) the enthalpy term could dominate and the equilibrium constant would then favour ${ }^{3} \mathrm{t}$. Blue shifts in the wavelength of the triplet-triplet absorption maxima of stilbene ${ }^{44}$ and nitrostilbenes ${ }^{5,6}$ when the medium is changed from a liquid solvent (e.g. benzene at room temperature) to frozen solution (e.g. EPA or hydrocarbon glass at $77 \mathrm{~K}$ ), have in fact been suggested to imply that the stilbene triplets are more twisted in the liquid state ${ }^{5,6,44}$. Since deuterium isotope effects on the decay rate constants of stilbene triplets provide strong evidence supporting a transoid geometry in rigid media, primarily owing to a medium imposed inhibition of ${ }^{3} \mathrm{t} \rightarrow{ }^{3} \mathrm{p}$ torsional displacement ${ }^{2}$, the assignment of the blue shifts to changes in geometry is probably accurate. It would be of interest to determine whether such shifts can be observed as the temperature is lowered in media which remain fluid throughout the temperature range (e.g. isopentane or propane).

\section{The energetics of twisting in the stilbene triplet state}

A possible energy diagram for twisting about the central bond in the stilbene triplet state which is consistent with all presently available spectroscopic, photochemical and quenching data is proposed in Figure $7^{49}$. The curve in dashed lines is based on assumed free energy differences $\left(\Delta F \simeq \Delta F_{15}\right)$ and accounts for the results of stilbene triplet interactions with quenchers. The free energy minimum is purposely displaced toward the cis side of $90^{\circ}$ because the natural decay ratio in solution, $\alpha /(1-\alpha)$, favours decay to the cis ground state as does the quenching interaction with tert-butyl nitroxide. A shallow minimum at the twisted configuration is shown since the deactivation of stilbene triplets by oxygen to either ground state isomer, suggests that the $22 \mathrm{kcal} / \mathrm{mol}$ energy gap required in equation 10 , provided ${ }^{1} \Delta_{\mathrm{g}}$ oxygen is formed, is achieved with nearly the same probability by torsional displacements towards either transoid or cisoid geometries. The dashed curve is flat towards the trans side and rises steeply towards the cis side since the larger energy gap required for excitation transfer to azulene $\left(E_{\mathrm{T}}=30\right.$ $\mathrm{kcal} / \mathrm{mol}$ ) is apparently achieved only by torsional displacement toward 
transoid geometries. In this connection we recall that stilbene triplet dynamics within donor-acceptor cages (or exciplexes) have previously been considered in discussing the interaction of the stilbenes with low triplet energy donors, $E_{\mathrm{T}} \lesssim 48 \mathrm{kcal} / \mathrm{mol}^{1,16,17}$. Since in such systems the triplet energies of donor and acceptor are balanced, electronic energy may remain with either partner upon diffusive separation. The experimental observations

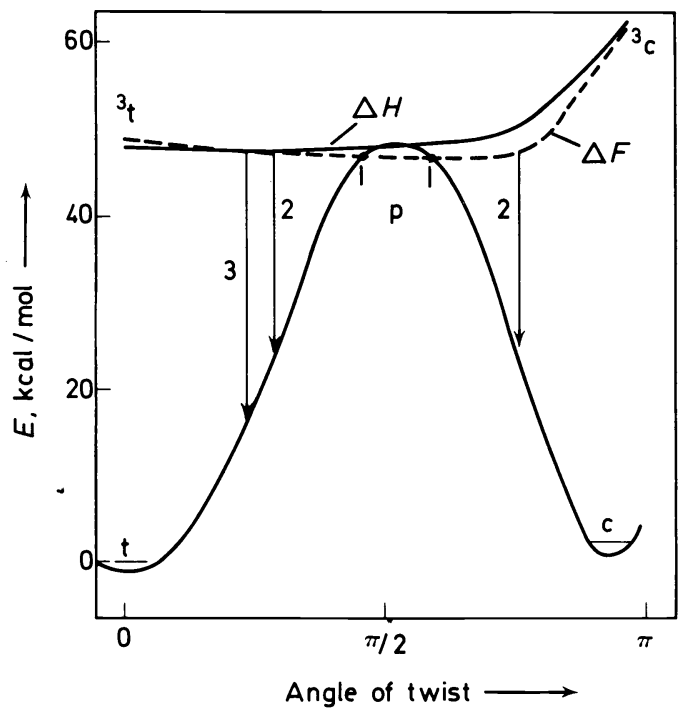

Figure 7. Possible energy diagram for twisting about the central bond of stilbene in the ground state and in the lowest triplet state (see text). The points labelled 1 may represent geometries of high natural decay probability. Lines 2 represent excitation transfer to oxygen and line 3 represents excitation transfer to azulene.

show that the excitation remains most often with the stilbene partner although the ${ }^{3} \mathrm{t}$ to ground state donor transfer is exothermic (consider, for example, benzanthrone, $E_{\mathrm{T}} \simeq 46 \mathrm{kcal} / \mathrm{mol}^{1,3}$ or 9,10 -dichloroanthracene, $\left.E_{\mathrm{T}}=40.2 \mathrm{kcal} / \mathrm{mol}^{16}\right)$. The speculation that such results reflect a relatively long residence time for the stilbene partner of the donor-acceptor pair in ${ }^{3} \mathrm{p}$ rather than ${ }^{3} \mathrm{t}$ geometries ${ }^{1}$ is clearly borne out by the present study.

Also shown in Figure 7 is a solid line for the triplet state which is tentatively based on $\Delta H_{15}$ and approximates the expected changes in potential energy as the central torsional angle is changed. This curve is consistent with the functioning of trans- and cis-stilbene as vertical and non-vertical acceptors of triplet excitation, respectively.

\section{THE POSITIONAL DEPENDENCE OF THE HEAVY ATOM EFFECT ON BROMOSTILBENE PHOTOISOMERIZATION}

Azulene quenching experiments have been used in determining the extent to which stilbene triplets participate in cis-trans photoisomerization following direct stilbene excitation ${ }^{4}$. On the basis of these experiments it has been concluded that at room temperature intersystem crossing is a negligible decay channel in the stilbenes ${ }^{4}$, but becomes a significant process in para 
substituted stilbenes provided that the substituent is capable of enhancing intersystem crossing. We have reported preliminary observations suggesting intersystem crossing enhancement in the $p$-bromostilbenes ${ }^{1}$, and Bent and Schulte-Frohlinde have published similar results for several $p$-nitrostilbenes $^{5,6}$. Since application of the azulene method as a mechanistic probe in stilbene photoisomerization has aroused some controversy ${ }^{50,51}$ it is important to note that by employing laser flash-kinetic spectroscopy Bent and Schulte-Frohlinde have provided direct confirmation of the validity of the method.

A substantial bromine substituent effect was revealed by comparison of the temperature dependence of fluorescence, $\Phi_{f}$, and isomerization, $\Phi_{t \rightarrow c}, \Phi_{c \rightarrow t}$, quantum yields for the stilbenes and the $p$-halostilbenes ${ }^{52-54}$. Increasing the temperature in the parent hydrocarbon from $-180^{\circ}$ to $25^{\circ}$ increases $\Phi_{t \rightarrow c}$ while decreasing $\Phi_{f}$, these two processes being coupled throughout the temperature range ${ }^{5-56}$. In $p$-bromostilbene $\Phi_{t \rightarrow c}$ is reported to be temperature independent while $\Phi_{\mathrm{f}}$ increases modestly as the temperature is decreased $^{53,53}$. The temperature independence of $\Phi_{c \rightarrow t}$ in the systems studied has revealed no difference in behaviour between excited cis-stilbene and its halogen substituted derivatives ${ }^{52,54}$. However, halogen substitution, especially in the meta position, has been reported to lower $\Phi_{c \rightarrow t}$ significantly $52,54,57$ and it has been suggested that enhanced spin-orbit coupling induces very rapid ${ }^{1} \mathrm{c} \rightarrow{ }^{3} \mathrm{c} \rightarrow{ }^{0} \mathrm{c}$ decay ${ }^{57,58}$. This suggestion contradicts the commonly expressed view ${ }^{1,6}$ that, with the exception of a usually minor cyclization component from ${ }^{1} \mathrm{c}$ to dihydrophenanthrene ${ }^{59}$ (DHP), torsion about the central bond to twisted geometries, ${ }^{1} \mathrm{p}$ and ${ }^{3} \mathrm{p}$, is the major decay mode of cisexcited states. The low $\Phi_{c \rightarrow t}$ values were obtained by u.v. analysis of stilbene mixtures resulting from excitation with $313 \mathrm{~nm}$ radiation ${ }^{57}$. Accurate measurements are difficult to make under these conditions since at this wavelength the extinction coefficients of the trans isomers are five to ten times larger than those of the cis isomers and photostationary states are generally $\sim 90$ per cent cis. Consequently, the low quantum yields could reflect analytical inaccuracy and/or failure to correct observed trans $\rightarrow$ cis conversions for large back reaction contributions. These considerations prompted the work described in the final part of this paper.

\section{Photoisomerization quantum yields}

Irradiations were carried out at $30^{\circ}$ in a merry-go-round apparatus using $313 \mathrm{~nm}$ light in the presence and in the absence of visible light absorbed by the DHPs. We have utilized larger stilbene concentrations in $n$-pentane and g.l.p.c. analyses and have made appropriate back-reaction corrections ${ }^{60}$. Large cis-trans conversions due to bromine atom catalysis were avoided by including mossy zinc in the bottom of the irradiation ampoules ${ }^{61}$. The benzophenone-sensitized photoisomerization of cis-1,3-pentadiene was used for actinometry ${ }^{60}$. Preliminary quantum yields obtained in our work ${ }^{62}$ are shown in Table 3 along with corresponding values reported by Krüger and Lippert ${ }^{57}$ and Malkin and Fischer ${ }^{52}$. Simultaneous visible irradiation does not affect trans $\rightarrow$ cis quantum yields but appears to increase cis $\rightarrow$ trans quantum yields for the bromostilbenes. This wavelength effect is not fully understood and will be investigated further. It is most likely related to 


\section{JACK SALTIEL ET AL.}

excitation of DHPs which form only from the cis isomers. While our observations indicate a modest decrease in $\Phi_{c \rightarrow t}$ for $m, m^{\prime}$-dibromostilbene, they do not show the dramatic decrease which was reported previously ${ }^{57}$. Furthermore, Table 3 shows that we find trans isomers to be significant components at photostationary states obtained by exciting at $313 \mathrm{~nm}$ and even more so when excitation is at $254 \mathrm{~nm}$ where cis and trans isomers have more nearly similar extinction coefficients (e.g. for $m, m^{\prime}$-dibromostilbene $\varepsilon_{\mathrm{c}} / \varepsilon_{\mathrm{t}}$ is 0.157 and 1.67 at 313 and $254 \mathrm{~nm}$, respectively) ${ }^{62}$.

Table 3. Isomerization quantum yields and photostationary compositions

\begin{tabular}{llllc}
\hline \multicolumn{1}{c}{ Compound } & \multicolumn{1}{c}{$\Phi_{\mathrm{t} \rightarrow \mathrm{c}}$} & \multicolumn{1}{c}{$\Phi_{\mathrm{c} \rightarrow \mathrm{t}}$} & \multicolumn{1}{c}{$\%[\mathrm{t}]_{\mathrm{s}}$} & $\%[\mathrm{t}]_{\mathrm{s}} \dagger$ \\
\hline Stilbene $\ddagger$ & $0.52(0.50)$ & $0.33(0.35)$ & $8.3(7)$ & 52 \\
$p$-Bromostilbene $\ddagger$ & $0.54(0.35)$ & $0.4(0.16)$ & $11.0(-)$ & 62 \\
$m$-Bromostilbene§ & $0.53(0.46)$ & $0.33(0.18)$ & $11.4(6)$ & 59 \\
$m, m^{\prime}$-Dibromostilbene $\S$ & $0.57(0.53)$ & $0.22(<0.05)$ & $8.0(\sim 0)$ & 69 \\
\hline
\end{tabular}

$\dagger$ These photostationary states are attained using $254 \mathrm{~nm}$ excitation.

¥ Values in parentheses are from reference 52 .

$\S$ Values in parentheses are from reference 57.

The benzophenone-sensitized photoisomerizations of the stilbenes in Table 3 give nearly identical photostationary states, $\sim 40$ per cent trans, see Table 4, which are approached at the same rate under identical conditions. Thus, sensitized cis $\rightarrow$ trans quantum yields are not diminished by bromine substitution and the postulated rapid ${ }^{3} \mathrm{c} \rightarrow \mathrm{c}$ decay $^{57}$ is ruled out.

Table 4. Azulene effects on photoisomerizations of stilbenes in $n$-pentane at $30^{\circ}$

\begin{tabular}{lcrl}
\hline \multicolumn{1}{c}{ Compound } & $r_{\mathrm{d}}^{\dagger}$ & $r_{\mathrm{s}} \ddagger$ & $\%[\mathrm{t}]_{\mathrm{s}} \S$ \\
\hline Stilbene & 14 & 304 & 37.0 \\
$p$-Bromostilbene & 31 & 56 & 39.6 \\
$m$-Bromostilbene & 12 & 140 & 45.3 \\
$m, m^{\prime}$-Dibromostilbene & 23 & 400 & 37.5 \\
\hline
\end{tabular}

† Slope/intercept ratios for excitation at $313 \mathrm{~nm}$; similar results were obtained at $254 \mathrm{~nm}$.

$\ddagger$ Benzophenone-sensitization.

$\S$ Photostationary states for benzophenone-sensitization in the absence of azulene, $\%[\mathrm{t}]_{\mathrm{s}}=100 \alpha$.

A possible explanation for the decrease in $\Phi_{c \rightarrow t}$ under direct excitation conditions is that it does not involve heavy-atom-enhanced intersystem crossing but is due to the larger moments of inertia of the aryl groups which may tend to favour cyclization to DHP over torsional ${ }^{1} \mathrm{c} \rightarrow{ }^{1} \mathrm{p}$ displacement. Support for this suggestion is provided by preliminary observations which show that prolonged parallel irradiation at $313 \mathrm{~nm}$ of degassed pentane solutions of the stilbenes and the $m, m^{\prime}$-dibromostilbenes over mossy zinc give large amounts of dibromophenanthrene from the latter ( 50 per cent, identification based on g.l.p.c. retention time) and very little phenanthrene from the former (five per cent). 


\section{Intersystem crossing efficiencies from azulene effects}

The most interesting result which has emerged from our experiments in this area has been a marked positional dependence for the ability of bromine to influence spin-orbit coupling. This positional dependence is inferred from measurements of photostationary states for the direct and benzophenonesensitized photoisomerization in the presence of azulene. In all cases $([\mathrm{t}] /[\mathrm{c}])_{\mathrm{s}}$ ratios are found to be linearly dependent on azulene concentration. Slope/ intercept ratios for the direct and sensitized experiments, in $n$-pentane $r_{d}$ and $r_{\mathrm{s}}$, respectively, are shown in Table 4. Similar observations have been made in benzene where the differences in $r_{\mathrm{d}}$ and $r_{\mathrm{s}}$ are less pronounced owing to the large viscosity dependence for $r_{\mathrm{s}}$ values (see Table 1 and Figure 1 for the parent stilbene).

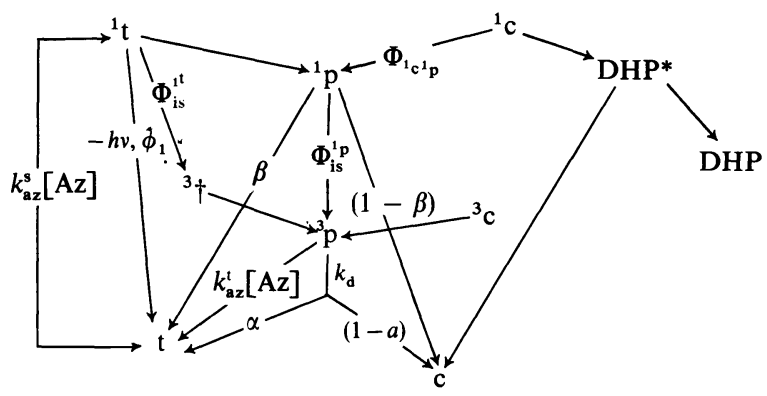

Scheme 3. Combination of singlet and triplets paths for stilbene photoisomerization

A general mechanism for stilbene isomerization following direct light absorption is shown in Scheme 3 , where $\Phi_{1_{\mathrm{c}^{1}} \mathrm{p}}$ is the quantum yield for twisted singlet formation from ${ }^{1} \mathrm{c}, \Phi_{\mathrm{is}}^{{ }_{1}}$ and $\Phi_{\mathrm{is}}^{{ }_{1}^{1} \mathrm{p}}$ are intersystem crossing quantum yields from ${ }^{1} \mathrm{t}$ and ${ }^{1} \mathrm{p}$, respectively, $k_{\mathrm{az}}^{\mathrm{s}}$ and $k_{\mathrm{az}}^{\mathrm{t}}$ are rate constants for azulene quenching of stilbene singlets and triplets, respectively, and other symbols have their usual meanings. The mechanism predicts that photostationary $[\mathrm{t}] /[\mathrm{c}]$ ratios for direct excitation should depend on azulene concentration according to equation 19 , where $K_{\mathrm{s}}=k_{\mathrm{az}}^{\mathrm{s}} \tau_{{ }_{\mathrm{t}}}$, and $K_{\mathrm{t}}=k_{\mathrm{az}}^{\mathrm{t}} / k_{\mathrm{d}}=\alpha r_{\mathrm{s}}$. In the

$$
\begin{aligned}
& ([\mathrm{t}] /[\mathrm{c}])_{\mathrm{s}}=\left(\varepsilon_{\mathrm{c}} / \varepsilon_{\mathrm{t}}\right) \Phi_{\mathrm{i}_{\mathrm{c}} \mathrm{p}}\left(1+K_{\mathrm{s}}[\mathrm{Az}]\right) \times \\
& \left\{\frac{\beta\left(1-\Phi_{\mathrm{is}}^{1 \mathrm{p}}\right)\left(1+K_{\mathrm{t}}[\mathrm{Az}]\right)+\Phi_{\mathrm{is}}^{\mathrm{p}}\left(\alpha+K_{\mathrm{t}}[\mathrm{Az}]\right)}{\left(1-\Phi_{\mathrm{f}}\right)(1-\alpha)+\left(1-\Phi_{\mathrm{is}}^{1 \mathrm{t}}-\Phi_{\mathrm{f}}\right)\left(1-\Phi_{\mathrm{is}}^{\mathrm{h}}\right)\left\{\alpha-\beta+(1-\beta) K_{\mathrm{t}}[\mathrm{Az}]\right\}}\right\}
\end{aligned}
$$

absence of intersystem crossing, $\Phi_{\text {is }}^{1} \sim \Phi_{\text {is }}^{1 t}=0$, equation 19 reduces to equation 20 so that $r_{\mathrm{d}}=K_{\mathrm{s}}$. If, on the other hand, intersystem were the only pathway leading to isomerization, equation 19 would reduce to equation 21

$$
\begin{gathered}
([\mathrm{t}] /[\mathrm{c}])_{\mathrm{s}}=\frac{\varepsilon_{\mathrm{c}}}{\varepsilon_{\mathrm{t}}} \frac{\Phi_{1_{\mathrm{c}} \mathrm{p}}}{1-\Phi_{\mathrm{f}}} \frac{\beta}{1-\beta}\left(1+K_{\mathrm{s}}[\mathrm{Az}]\right) \\
([\mathrm{t}] /[\mathrm{c}])_{\mathrm{s}}=\frac{\varepsilon_{\mathrm{c}}}{\varepsilon_{\mathrm{t}}} \frac{\Phi_{1_{\mathrm{c}} \mathrm{p}}}{1-\Phi_{\mathrm{f}}}\left(1+K_{\mathrm{s}}[\mathrm{Az}]\right) \frac{\left(\alpha+K_{\mathrm{t}}[\mathrm{Az}]\right)}{(1-\alpha)}
\end{gathered}
$$


and the dependence on azulene would deviate somewhat from linearity, with $r_{\mathrm{d}}>r_{\mathrm{s}}{ }^{4}$.

Using Förster's theory for long-range singlet energy transfer a value of $K_{\mathrm{s}}=15 \mathrm{M}^{-1}$ can be calculated which is based on the spectral overlap between trans-stilbene fluorescence and azulene absorption ${ }^{1,4}$. In the case of the bromostilbenes a smaller $K_{\mathrm{s}}$ value could result if $\tau_{\mathrm{t}_{\mathrm{t}}}$ is decreased owing to enhanced intersystem crossing. Examination of the data in Table 4 shows that $K_{\mathrm{s}} \simeq r_{\mathrm{d}}$ (equation 20) for the cases of the stilbenes, the $m$-bromostilbenes and the $m, m^{\prime}$-dibromostilbenes but that $r_{\mathrm{d}}$ approaches $r_{\mathrm{s}}$ in the case of the $p$-bromostilbenes. It follows that bromine enhances intersystem crossing when bonded at a para position, but is completely $\dagger$ ineffective in this respect when bonded at a meta position. This striking positional dependence of radiationless heavy-atom induced spin-orbital coupling is to our knowledge unprecedented. The relatively modest positional dependence observed in dibromonaphthonorbornenes is not analogous since substitution was not directly on a carbon of the $\pi$-system as in the bromostilbenes ${ }^{63}$.

The $p$-bromostilbene data correspond to neither of the limiting cases described by equations 20 and 21 and require the use of equation 19 for their explanation. A fitting of the data to equation 19 can be accomplished by (1) using $K_{t}$ and $\alpha$ from the benzophenone-sensitized experiments, (2) assuming $\Phi_{\mathrm{c}^{1} \mathrm{p}}=1-\Phi_{\mathrm{DHP}} \simeq 0.9$ as in the case of the unsubstituted stilbene ${ }^{59}$, and (3) estimating the quantum yield of the non-activated process competing with fluorescence, presumably $\Phi_{\text {is }}^{1 t}$, from the temperature dependence ${ }^{52,53}$ of trans-p-bromostilbene fluorescence ${ }^{1,64}$. The values of $\Phi_{\text {is }}^{1 \text { t }}$ obtained from the fluorescence data suggest that approximately 30 per cent of trans-pbromostilbene singlets intersystem cross at $30^{\circ}$. The functioning of this additional decay channel is expected to decrease the lifetime of trans singlets by about 30 per cent resulting in $K_{\mathrm{s}} \simeq 10 \mathrm{M}^{-1}$ for trans-p-bromostilbene ( $\sim 70$ per cent of $14 M^{-1}$, see Table 4). The value of $\beta$ can be calculated from $([\mathrm{t}] /[\mathrm{c}])_{\mathrm{s}}$ at zero azulene concentration using the measured value of $\varepsilon_{\mathrm{c}} / \varepsilon_{\mathrm{t}}$ at the excitation wavelength. An appropriate value of $\Phi_{\text {is }}^{1 p}$ is finally chosen which fits equation 19 to the experimental photostationary states at all azulene concentrations. Observed and calculated photostationary compositions for the direct photoisomerization of the $p$-bromostilbenes in $n$-pentane employing 313 and $254 \mathrm{~nm}$ excitation conditions are shown in Table 5. It can be seen that excellent agreement with the experimental observations is obtained by using $\Phi_{\text {is }}^{1}$ values of 0.28 and 0.48 for the 313 and $254 \mathrm{~nm}$ experiments, respectively. The assumption of the same $\Phi_{\text {is }}^{i_{t}}$ value at the two wavelengths is probably justified since preliminary measurements indicate that $\Phi_{\mathrm{f}}$ of trans$p$-bromostilbene is also the same. The difference in the $\Phi_{\text {is }}^{1{ }^{1}}$ values may suggest that Scheme 3 should include an additional intersystem crossing step which operates following $254 \mathrm{~nm}$ excitation. Speculation concerning such a step would be premature since the difference may not be significant when compared with the experimental uncertainty of the measurements.

Barring large substituent effects on the effective rate constant for triplet

$\dagger$ The value of $r_{\mathrm{d}}=23 \mathrm{M}^{-1}$ for the $m, m^{\prime}$-dibromostilbenes may indicate a tiny intersystem crossing component in this case, but may be within experimental uncertainty of $15 \mathrm{M}^{-1}$. A value of $r_{\mathrm{d}}=12 \mathrm{M}^{-1}$ was obtained for this compound in benzene. Cf., however, note added in proof. 
excitation transfer to azulene (see equation 17), the relative magnitude of the $r_{\mathrm{s}}$ values in Table 4 should be related approximately to the inverse of the rate constant for intersystem crossing from ${ }^{3} \mathrm{p}$. Examination of these values suggests a pronounced enhancement of $k_{\mathrm{d}}$ for bromine substitution at the para position and little or no enhancement when bromine is bonded at a meta position.

Table 5. The azulene effect on the direct photoisomerization of the $p$-bromostilbene in $n$-pentane at $30^{\circ}$

\begin{tabular}{|c|c|c|c|c|c|}
\hline \multicolumn{3}{|c|}{$313 \mathrm{~nm}$} & \multicolumn{3}{|c|}{$254 \mathrm{~nm}$} \\
\hline$[\mathrm{Az}] \times 10^{3} \mathrm{M}$ & $\%[\mathrm{t}]_{\mathrm{cal}}{ }^{\dagger}$ & $\%[t]_{\text {obs }}$ & {$[\mathrm{Az}] \times 10^{3} \mathrm{M}$} & $\%[t]_{\text {calc }} \ddagger$ & $\%[t]_{\text {obs }}$ \\
\hline 0 & 11.0 & $11.0 \S$ & 0 & 61.8 & $61.8 \S$ \\
\hline 5.0 & 12.4 & 12.4 & 7.6 & 67.9 & 67.8 \\
\hline 9.0 & 13.5 & 13.7 & 15.1 & 72.3 & 73.1 . \\
\hline 12.5 & 14.5 & 14.4 & 22.7 & 75.8 & 76.0 \\
\hline 20.0 & 16.5 & 16.5 & 30.3 & 78.6 & 78.5 \\
\hline 25.0 & 17.8 & 18.0 & & & \\
\hline
\end{tabular}

$\dagger$ From equation 19 using $\Phi_{i s}^{1} p=0.28, \Phi_{i s}^{1 t}=0.31, \alpha=0.396, \beta=0.462$.

$\ddagger$ From equation 19 using $\Phi_{\mathrm{is}}^{\mathrm{s}}=0.48, \Phi_{\mathrm{is}}^{\mathrm{is}}=0.31, \alpha=0.396, \beta=0.479$.

$\S$ These values are those predicted by the intercepts of the azulene plots.

The positional dependence is probably related to the difference between meta and para coefficients of the highest occupied and lowest unoccupied MOs of stilbene, $c_{m}=0.0791$ and $c_{p}=0.3138$ in the Hückel approximation. Spin-orbit coupling occurs via interaction with upper $\sigma \pi^{*}$ and $\pi \sigma^{*}$ singlet and triplet states ${ }^{65,66}$. Qualitatively, spin-orbit coupling matrix elements can be approximated by one centre term and can be reduced to a centre by centre sum of the $\pi$-electron coefficients times the approximate $\sigma$ orbital coefficients $^{66}$. If, as in the case of bromine substitution the centre bearing bromine dominates then the expression describing the probability of spinorbital coupling will be dominated by the appropriate $\pi$ and $\sigma$ MO coefficients at that centre. ${ }^{66} \mathrm{It}$ is sensible, therefore, that since the $\pi$-coefficient of stilbene is nearly zero at the meta position, substitution at that position should cause no enhancement in the rate constants for intersystem crossing.

\section{ACKNOWLEDGEMENTS}

This work was supported in part by the National Science Foundation, Grant No. GP-24265 and by the Alfred P. Sloan Foundation. J. S. was an Alfred P. Sloan Fellow, 1971-1973, and E. D. M. was a National Institutes of Health Predoctoral Fellow, 1965-1969. We thank Dr Alan Sykes and $\mathrm{Mr}$ David E. Townsend for carrying out initial experiments with our flashphotolysis apparatus, and Professor DeLos F. DeTar for helping us apply his general non-linear least squares computer programme 'GENLSS' to the analyses of the flash-kinetic measurements. 


\section{JACK SALTIEL ET AL.}

\section{REFERENCES}

1 J. Saltiel, J. D’Agostino, E. D. Megarity, L. Metts, K. R. Neuberger, M. Wrighton and O. C. Zafiriou, Org. Photochem. 3, 1 (1973).

2 J. Saltiel, J. T. D'Agostino, W. G. Herkstroeter, G. Saint-Ruf and N. P. Buu-Hoï, J. Amer. Chem. Soc. 95, 2593 (1973).

3 G. S. Hammond, J. Saltiel, A. A. Lamola, N. J. Turro, J. S. Bradshaw, D. O. Cowan, R. S. Counsell, V. Vogt and C. Dalton, J. Amer. Chem. Soc. 86, 3197 (1964).

4 J. Saltiel and E. D. Megarity, J. Amer. Chem. Soc. 91, 1265 (1969); 94, 2742 (1972) and references cited therein.

5 D. V. Bent and D. Schulte-Frohlinde, J. Phys. Chem. 78, 446 (1974).

6 D. V. Bent and D. Schulte-Frohlinde, J. Phys. Chem. 78, 451 (1974).

7 J. Saltiel, J. Amer. Chem. Soc. 89, 1036 (1967); 90, 6394 (1968).

8 W. G. Herkstroeter and G. S. Hammond, J. Amer. Chem. Soc. 88, 4769 (1966).

9 cis-Stilbene is said to be a non-vertical acceptor of triplet energy. For a discussion of the current interpretation of this process which is due to Bylina ${ }^{10}$ see reference 1. For an elaboration of Bylina's suggestion see reference 11.

10 A. Bylina, Chem. Phys. Letters, 1, 509 (1968).

11 S. Yamauchi and T. Azumi, J. Amer. Chem. Soc. 95, 2709 (1973).

12 (a) R. A. Caldwell and R. E. Schwerzel, J. Amer. Chem. Soc. 94, 1035 (1972);

(b) R. A. Caldwell and R. E. Schwerzel, J. Amer. Chem. Soc. 95.1382 (1973).

13 A. A. Lamola, Tech. Org. Chem. 14, 17 (1969).

14 P. Borrell and H. H. Greenwood, Proc. Roy. Soc. (London), A, 298, 453 (1967).

15 Cf. also references $16-18$.

16 D. Gegiou, K. A. Muszkat and E. Fischer, J. Amer. Chem. Soc. 90, 12 (1968).

17 A. Bylina and Z. R. Grabowski, Trans. Faraday Soc. 65, 458 (1969).

18 S. Sharafy and K. A. Muszkat, J. Amer. Chem. Soc. 93, 4119 (1972).

19 J. Saltiel, H. C. Curtis, L. Metts, J. W. Miley, J. Winterle and M. Wrighton, J. Amer. Chem. Soc. 92,410 (1970).

20 P. M. Rentzepis, Chem. Phys. Letters, 3, 717 (1969).

21 D. G. Whitten and Y. J. Lee, J. Amer. Chem. Soc. 94, 9142 (1972).

22 R. S. H. Liu, J. Amer. Chem. Soc. 90, 1899 (1968).

${ }^{23}$ K. S. Y. Lau, R. O. Campbell and R. S. H. Liu, Mol. Photochem. 4, 315 (1972).

24 P. J. Wagner and I. Kochevar, J. Amer. Chem. Soc. 90, 2232 (1968).

25 J. Saltiel, A. D. Rousseau and A. Sykes, J. Amer. Chem. Soc. 94, 5903 (1972).

26 J. Saltiel, A. D. Rousseau and B. Thomas, unpublished observations.

27 A. D. Osborne and G. Porter, Proc. Roy. Soc. (London), A, 284, 9 (1965).

${ }^{28}$ N. J. Turro, N. E. Schore, H.-C. Steinmetzer and A. Yekta, J. Amer. Chem. Soc. 96, 1936 (1974) and references cited therein.

29 J. Saltiel, P. Shannon and A. Uriarte, unpublished results.

30 G. Jackson and R. Livingston,J. Chem. Phys. 35. 2182 (1961).

31 A. A. Frost and R. G. Pearson, Kinetics and Mechanism, p 152. Wiley: New York (1953).

32 D. F. DeTar, Computer Programs for Chemistry, 4, 71 (1972).

33 W. R. Ware and J. S. Novros, J. Phys. Chem. 70, 3247 (1966).

34 E. Clar, J. Chem. Soc. 1823 (1950).

35 A. F. Vaudo and D. M. Hercules, J. Amer. Chem. Soc. 92, 3537 (1970).

36 A. F. Vaudo and D. M. Hercules, J. Amer. Chem. Soc. 93, 2599 (1971).

${ }^{37}$ P. Lechtken, A. Yetka and N. J. Turro, J. Amer. Chem. Soc. 95, 3027 (1973), and references cited therein.

38 N. J. Turro, P. Lechtken, G. Schuster, J. Orell, H.-C. Steinmetzer and W. Adam, J. Amer. Chem. Soc. 96, 1627 (1974).

39 M. Yokota and O. Tannimoto, J. Phys. Soc. Japan, 22, 779 (1967).

40 A. R. Watkins, Chem. Commun. 634 (1973).

41 J. Saltiel, A. D. Rousseau and D. E. Townsend, unpublished results.

42 J. Saltiel and J. T. D'Agostino, J. Amer. Chem. Soc. 94, 6445 (1972).

43 Cf., however, R. G. Bennett and P. J. McCartin, J. Chem. Phys. 44, 1969 (1966);

E. C. Lim, J. D. Laposa and J. M. H. Yu, J. Mol. Spectrosc. 19, 412 (1966).

44 F. S. Dainton, E. A. Robinson and G. A. Salmon, J. Phys. Chem. 76, 3897 (1972).

45 J. Saltiel and B. Thomas, J. Amer. Chem. Soc. 96. 5660 (1974).

46 J. Saltiel, D. E. Townsend and A. Sykes, J. Amer. Chem. Soc. 95, 5968 (1973). 
47 For a related case see R. A. Caldwell, J. Amer. Chem. Soc. 92, 3229 (1970).

48 Part of the discrepancy may be due to the somewhat low assumed value of $k_{\text {dif }}=5.0 \times$ $10^{9} M^{-1} \mathrm{~s}^{-1}$ in benzene on which most rate constants in reference $12 \mathrm{~b}$ are based.

49 See reference 1 for a review of relevant data not discussed in this paper.

50 E. Fischer, Mol. Photochem. 5, 227 (1973).

51 J. Saltiel, Mol. Photochem. 5, 231 (1973).

52 S. Malkin and E. Fischer, J. Phys. Chem. 68, 1153 (1964) and previous papers in this series.

53 H. Dyck and D. S. McClure, J. Chem. Phys. 36, 2326 (1962).

54 D. Gegiou, K. A. Muszkat and E. Fischer, J. Amer. Chem. Soc. 90,3907 (1968).

55 S. Sharafy and K. A. Muszkat, J. Amer. Chem. Soc. 93, 4119 (1971).

56 J. Saltiel and J. T. D'Agostino, J. Amer. Chem. Soc. 94, 6445 (1972).

57 K. Krüger and E. Lippert, Z. Phys. Chem., NF, 66, 293 (1969).

58 E. Lippert, Z. Phys. Chem., NF, 42, 125 (1964).

59 See, for example, K. A. Muszkat and E. Fischer, J. Chem. Soc. B, 662 (1967).

60 A. A. Lamola and G. S. Hammond, J. Chem. Phys. 43, 2129 (1965).

61 P. J. Kropp, T. H. Jones and G. S. Poindexter, J. Amer. Chem. Soc. 95, 5420 (1973).

62 J. Saltiel, D. W.-L. Chang and E. D. Megarity, J. Amer. Chem. Soc. 96, 6521 (1974).

${ }^{63}$ N. J. Turro, G. Kavarnos, V. Fung, A. L. Lyons Jr, and T. Cole Jr, J. Amer. Chem. Soc. 94, 1392 (1972).

${ }^{64}$ E. P. Kirby and R. F. Steiner, J. Phys. Chem. 74, 4480 (1970).

65 S. P. McGlynn, T. Azumi and M. Kinoshita, Molecular Spectroscopy of the Triplet State, p 269. Prentice-Hall: New Jersey (1969).

66 B. R. Henry, personal communication.

\section{NOTE ADDED IN PROOF}

Recent observations by D. W. L. Chang and A. Marinari in our laboratory have led us to conclude that intersystem crossing yields based on photostationary states for the direct photoisomerization of the stilbenes in the presence of azulene are not reliable. Much larger azulene effects are observed on the initial $\mathrm{t} \rightarrow \mathrm{c}$ quantum yields for the direct photoisomerization. A preliminary analysis of these data together with the temperature dependence of the fluorescence yields of the trans-stilbenes in $n$-pentane gives the quantum yields shown in Table 6. While a positional dependence of the heavy

Table 6. Revised preliminary intersystem crossing yields

\begin{tabular}{lcc}
\hline \multicolumn{1}{c}{ Compound } & $\Phi_{\mathrm{is}}^{1_{\mathrm{t}}}$ & $\Phi_{\mathrm{is}}^{1_{\mathrm{p}}}$ \\
\hline Stilbene & 0.00 & 0.34 \\
$p$-Bromostilbene & 0.31 & 1.00 \\
$m$-Bromostilbene & 0.16 & 0.86 \\
$m, m^{\prime}$-Dibromostilbene & 0.37 & 0.84 \\
\hline
\end{tabular}

atom effect is still apparent it is much more modest than had been previously supposed. 